\title{
DESIGN OF PASSIVE TRANSPORT NOISE BARRIER HAVING AREAS OF HIGH NOISE LEVEL FOR ACOUSTIC ENERGY HARVESTING
}

\author{
IVAN KRALOV \\ Technical University of Sofia, Department of Mechanics, \\ 8, St. Kliment Ohridski Blvd, 1756 Sofia, Bulgaria, \\ e-mails: kralov@tu-sofia.bg
}

\begin{abstract}
Noise reflected from traffic barriers usually leads to increasing of the noise level excited by the moving vehicles and trains due to combined effect of source and reflected noise. The number of people affected by this effect usually is many times bigger than number of affected people behind the barrier. One of the existed solutions is use of expensive combined noise barriers of high degree of absorption. In this study a new passive transport noise barrier having high level of reduction of the reflected noise is treated. Acoustic efficiency is done by appropriate design of the barrier profile performing source and reflected acoustic waves interference in small volumes near the barrier. Also, these volumes of intensive acoustic waves interference near the barrier have frequency bands of high acoustic energy levels and are suitable for energy harvesters. An analysis of the acoustic energy distribution near the studied barrier is done using numerical simulation and experimental validation in this work. Based on the results analysis, some conclusions about usage and positioning of sound energy harvesters are made.

Keywords: acoustic energy harvesting, active noise control, passive transport noise barriers.
\end{abstract}

\section{INTRODUCTION}

Reduction of the acoustic noise reflects directly on the people health and environment protection. Three main ways are used to reduce the acoustic noise: source sound power reduction, influence upon the noise radiation between the source and the recipient, and noise insulation of the working/living place. In many cases application of the second method includes typical solution for reduction of noise propagation by different types of barriers, absorbers or active noise control solutions. The enormous number of patented solutions

DOI: 10.7546/EngSci.LVII.20.01.04 
and recent research works and publications in this area prove the actuality of this research field [1-8]. Meeting the noise level standards requires expensive and sophisticated solutions. The noise barriers are mainly divided into passive, active and combined. Passive solutions mainly block the spreading of sound waves and decrease the noise levels by reflecting acoustic waves $[1$, 9-14]. On the other hand, active methods input additional energy that is used to eliminate the acoustic energy of the source by propagation of sound waves of opposite phase [9], as well as a conversion of the sound energy to electricity could be included $[12,15-17]$. Combined solutions include combination of the previously mentioned ones.

The relatively low cost of manufacturing, assembly and maintenance due to the use of a few materials only, as well as the use of assemblies of equally shaped elements are the main advantages of the passive noise barriers. This is proved by the huge number of patented solutions [5-9, 18]. A lot of studies show good acoustic efficiency of these barriers regarding the relation of the noise level behind the barrier with respect to the source noise level $[1,11,14]$.

Combined types of noise barriers are used in case of higher level of noise reduction needed. They usually have additional closed space for spreading the sound waves and/or additional absorption materials are used. This type of barriers is relatively more expensive for manufacturing, assembly and maintenance. Its advantage is better noise energy absorption $[1,9]$.

For the noise excited by the transport flows, it is very essential to reduce it not only behind the barrier, but also the barrier reflected noise to be reduced. Noise reflected from traffic barriers usually leads to increasing of the noise level excited by the automobiles and trains. This effect insists transport noise barriers to be designed for effective noise reduction behind them as well as to produce small level of reflected noise in road direction. This will affect in better noise environment for many passengers especially in case of busy traffic in rush hours. People affected by this effect are usually far more than the affected ones behind the barrier. One of the existed solutions is the use of the above mentioned expensive combined noise barriers of high degree of absorption $[1,9]$.

Nowadays there were designed different technologies and solutions for transferring the wave energy into electric one. Some solutions allow use of thin shaped plates from specific materials to convert vibrational energy (including noise energy) to electricity [12, 19-27]. These solutions could be implemented in the passive noise barrier walls. It will lead to a new combined noise barrier of better efficiency. The aim of this research study is to present a design of passive transport noise barriers having areas of high noise level for acoustic en- 
ergy harvesting. The reflected noise level is done by appropriate design of the barrier profile performing source and reflected acoustic waves interference in small volumes near the barriers. The barrier construction, made by repeated equally profiled elements, allows even the existence of acoustic bandgaps especially behind the barrier. A numerical model and analysis will be made to estimate the presence of high level of sound energy areas for implementing the noise-electricity conversion solutions.

\section{DESIGN OF A TRANSPROT ACOUSTIC BARRIER UNDER STUDY}

To reach the aim defined above, it is used the acoustic noise barrier construction, shown in Fig. 1 [14]. Noise insulating panels are made of non-metal materials. They have a half-tube like form arranged one over the other, and all their concave arc profiles have one and the same direction - to the sound source waves. The essential characteristic of the barrier construction is that the concave profile is exactly semi-cylindrical and the border area between two neighbor channels is a sharp edge. These channels are arranged into H-shaped metal columns fixed to the ground. The channels are fixed vertically to the columns through non-metal semi-disks of external diameter, equal to the internal diameter of the channels. The semi-disks are fixed to the columns by bolt or stud connection. More could be found in [14]. The main advantages of this barrier are relatively high level of reflected noise reduction, as well as relatively low costs of manufacturing, transportation, assembly and maintenance. In addition, there are areas of noise wave interference in front of the barrier and close to it. Investigation of the sound energy distribution around the barrier will answer for the best places for noise-electricity convertors arrangement.
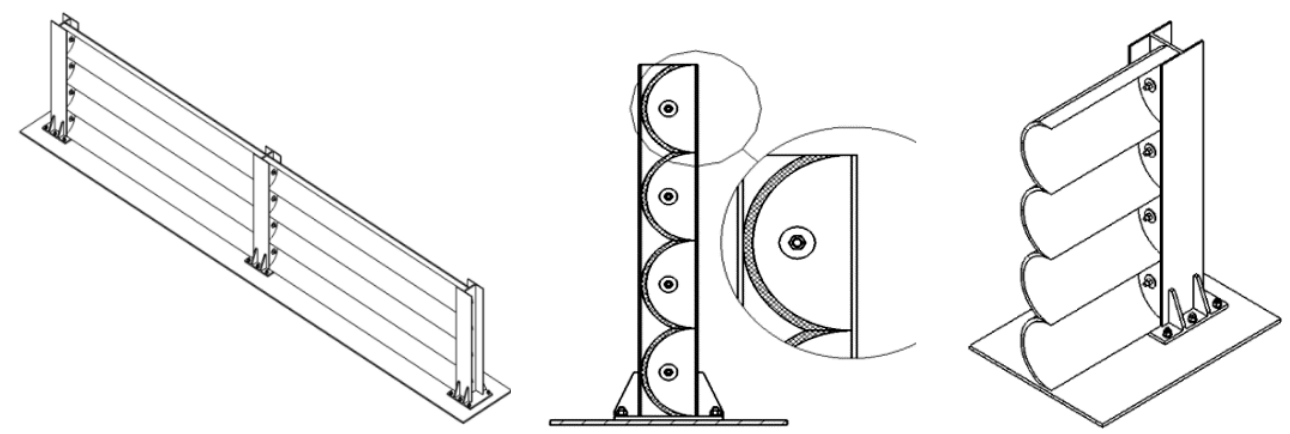

Fig. 1. New acoustic barrier design [14] 
This barrier makes the interference between the source and reflected sound waves to be close to the barrier. In this way the noise reduction by converting sound energy into heat will have lower effect over the noise level near the source. The degree of reflection depends mainly on the barrier material, sound waves frequency and acoustic pressure level. An important parameter of the barrier construction is the absence of reflecting surface, perpendicular to the source sound waves. The exact semi-cylindrical profile of the channels focuses reflected waves to interfere with the source waves inside the profile of the channels. This makes these volumes appropriate for placing the harvesting devices.

To achieve better performance, geometrical parameters of the barrier can be designed for specific cases. Varying the width of the profile and the radius of the channel according to the sound power and frequency is either a successful way of improving the acoustic efficiency of the barrier, or a way to focus the areas for noise-electricity conversion.

\section{SIMULATION, TEST AND ANALYSIS OF THE ACOUSTIC ENERGY DISTRIBUTION NEAR THE TREATED NOISE BARRIER}

The main investigation of the acoustic properties of new barrier is made by numerical simulation using a professional software for acoustics - COMSOL Multiphysics ${ }^{\circledR}$. Some of the numerical results are verified by a laboratory experiment using a scaled prototype of the barrier made.

\subsection{Experimental test of the scaled prototype of the new acoustic barrier}

To produce some laboratory experiments a scaled prototype of the barrier is made. The prototype is shown in Fig. 2(a) and its main parameters are given in Table 1. The test is shown in Fig. 2(b).

Table 1. Main parameters of the scaled prototype of the barrier under study

\begin{tabular}{|c|l|c|}
\hline No & \multicolumn{1}{|c|}{ Parameter } & Value \\
\hline 1 & Diameter of the channel profile (internal), $\mathrm{mm}$ & 76 \\
\hline 2 & Number of channels & 7 \\
\hline 3 & Width of the profiled channels, mm & 8 \\
\hline 4 & Material & HDPV \\
\hline 5 & Material density, $\mathrm{kg} / \mathrm{m}^{3}$ & 970 \\
\hline
\end{tabular}

Engineering Sciences, LVII, 2020, No. 1 


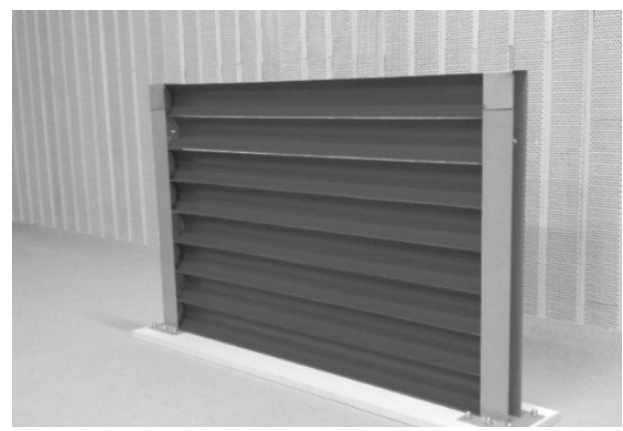

(a)

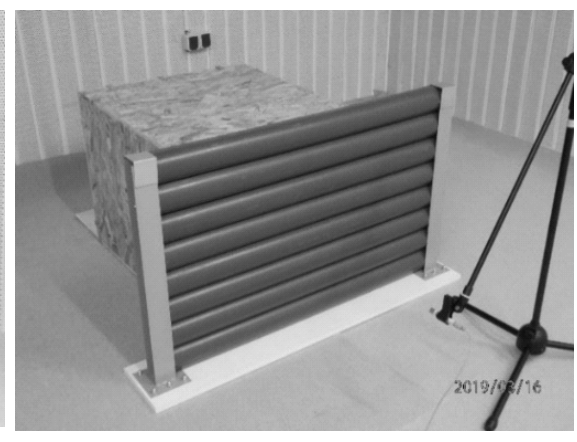

(b)

Fig. 2. (a) The scaled prototype of the new acoustic barrier;

(b) acoustic test of the prototype

The experiments were performed in a $40 \mathrm{~dB}$ semi-anechoic chamber. The noise level inside the chamber and the noise level at the receiving position (behind the barrier) were measured. The acoustic measurement and analyzing system PULSE 3560B of the Bruel \& Kaer equipped with the OEM software and microphone $4516 \mathrm{~A}$ is used. Before measurements, all the standard procedures for calibration and estimation of the environmental noise influence are proceeded. More information could be found in [13].

The results from the experiment are given in the Table 2. The following notations are used:

$L_{\text {fon }} \quad$ level of the surrounding noise, $\mathrm{dB}$;

$L_{S} \quad$ noise level of the source, $\mathrm{dB}$;

$L_{R P}(A) \quad A$-weighted noise level at the receiving point without barrier, dB;

Table 2. Results from experimental test of the scaled prototype of the barrier under study

\begin{tabular}{|c|c|c|c|c|}
\hline Octave, $\mathrm{Hz}$ & $L_{\text {fon }}, \mathrm{dB}$ & $L_{S}, \mathrm{~dB}$ & $L_{R P}(A), \mathrm{dB}$ & $L_{(R P, b a r)}(A), \mathrm{dB}$ \\
\hline $63 \mathrm{~Hz}$ & 19.7 & 67.9 & 48.2 & 41.8 \\
\hline $125 \mathrm{~Hz}$ & 22.1 & 68.1 & 54.3 & 47.2 \\
\hline $250 \mathrm{~Hz}$ & 23.0 & 68.2 & 58.9 & 51.2 \\
\hline $500 \mathrm{~Hz}$ & 19.6 & 68.2 & 62.8 & 49.1 \\
\hline $1000 \mathrm{~Hz}$ & 21.5 & 68.2 & 65.7 & 50.2 \\
\hline $2000 \mathrm{~Hz}$ & 16.7 & 68.2 & 66.4 & 46.6 \\
\hline $4000 \mathrm{~Hz}$ & 15.7 & 68.3 & 66.2 & 44.3 \\
\hline$E_{q}$ & 28.3 & 76.0 & 71.8 & 56.5 \\
\hline
\end{tabular}


$L_{(R P, b a r)}(A) \quad A$-weighted noise level at the receiving point with new barrier, dB;

$E_{q} \quad$ equivalent noise level for all the treated octaves, dB.

The results show an $A$-weighted noise level reduction in case of use of the barrier prototype between $16 \mathrm{~dB}$ and $22 \mathrm{~dB}$.

\subsection{Numerical simulation and test of the scaled prototype of the new acoustic barrier}

Numerical simulation of the same scaled barrier prototype is made in COMSOL Multiphysics ${ }^{\circledR}$. The process of model building, model mesh, type of analysis and boundary conditions are the same as in [13]. Comparative results of the new barrier acoustic efficiency are presented in Table 3 and in Fig. 3. Here the following new notations are used:

$L_{(R P, b a r, n)}(A) \quad A$-weighted noise level at the receiving point with new barrier, numerically found, $\mathrm{dB}$;

$L_{(R P, b a r, e)}(A) \quad A$-weighted noise level at the receiving point with new barrier, experimentally found, $\mathrm{dB}$.

Results show relatively good relevance of the experimental and numerical results for the scaled prototype of the new barrier acoustic efficiency. Differences are less than $30 \%$ for different octave bands which is appropriate for such basic research problem and is similar to the known results in the literature. Based on this conclusion next step of investigation is done.

Table 3. Comparative results from experimental and numerical test of the scaled prototype of the barrier under study [13]

\begin{tabular}{|c|c|c|c|c|}
\hline $\begin{array}{c}\text { Octave } \\
\mathrm{Hz}\end{array}$ & $\begin{array}{c}L_{(R P, \text { bar }, n)}(A) \\
\mathrm{dB}\end{array}$ & $\begin{array}{c}L_{(R P, \text { bar }, e)}(A) \\
\mathrm{dB}\end{array}$ & $\begin{array}{c}L_{(R P, \text { bar }, n)}(A)- \\
L_{(R P, b a r, e)}(A), \mathrm{dB}\end{array}$ & $\begin{array}{c}L_{(R P, b a r, n)}(A)- \\
L_{(R P, b a r, n)}(A), \%\end{array}$ \\
\hline 63 & 39.6 & 41.8 & -2.2 & $-5.3 \%$ \\
\hline 125 & 48.4 & 47.2 & 1.2 & $2.5 \%$ \\
\hline 250 & 47.1 & 51.2 & -4.1 & $-8.0 \%$ \\
\hline 500 & 56.7 & 49.1 & 7.6 & $15.5 \%$ \\
\hline 1000 & 59.1 & 50.2 & 8.9 & $17.7 \%$ \\
\hline 2000 & 54.6 & 46.6 & 8.0 & $17.2 \%$ \\
\hline 4000 & 53.7 & 44.3 & 9.4 & $21.2 \%$ \\
\hline
\end{tabular}

Engineering Sciences, LVII, 2020, No. 1 


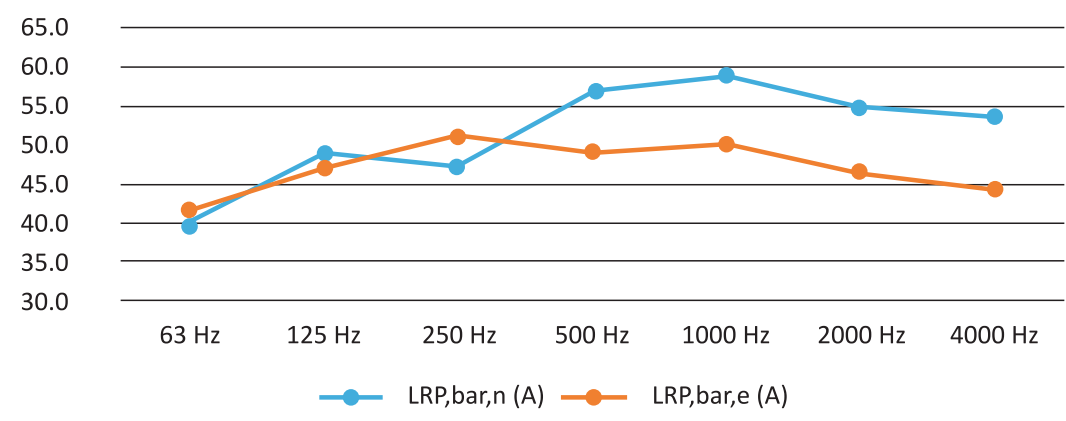

Fig. 3. Comparative results from experimental and numerical test of the scaled prototype of the barrier under study [13]

\subsection{Numerical investigation of the acoustic energy distribution near the barrier}

In this section an investigation of the noise level distribution on both the sides of the new acoustic barrier is presented in the vertical plain. The noise is emitted by a small source of spherical noise waves. The studied acoustic barrier as well as the environment around it are modelled as a "three-dimensional" 2D axisymmetric object [13]. For the present study a Frequency Acoustic Analysis-Pressure Acoustic, Frequency Domain is used. Again, the COMSOL Multiphysics ${ }^{\circledR}$ is used. The process of material parameters, model building,

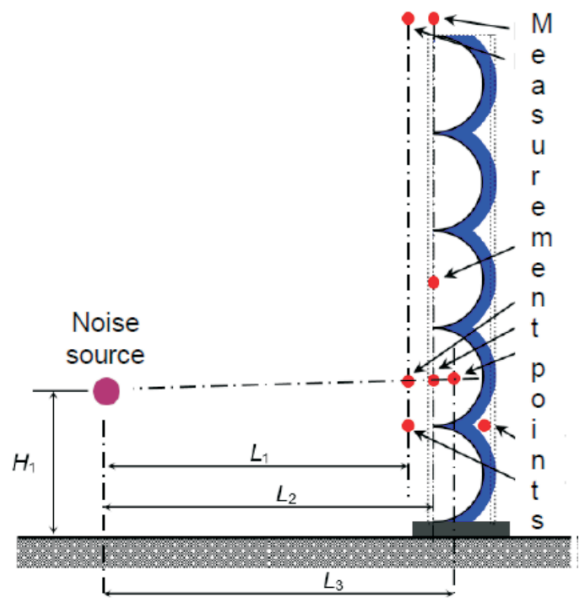

Fig. 4. Scheme of the investigation and measurement points 
model mesh, type of analysis and boundary conditions are the same as in [13].

Scheme of the investigation is presented in Fig. 4. The source $\mathrm{S}$ emits spherical waves. They reach the front surface of the barrier and reflect. In the near the barrier volumes both types of the waves (from the source and reflected ones) interfere. It is made a measurement of the noise levels at typical points and volumes in front of the barrier surface to find the presence of places of high-level acoustic energy. Figure 5 shows the numerical results for noise distribution in the vertical plane of the source at $1 \mathrm{kHz}$ and $2 \mathrm{kHz}$ without barrier. Figure 6 shows noise level distribution with barrier at frequency octave bands of $1000 \mathrm{~Hz}$ and $2000 \mathrm{~Hz}$, respectively.

To study the spatial distribution of the sound pressure around the barrier, a parametric numerical study was performed at different frequency ranges of the source, both in octave bands and in a wider "white noise" frequency range. For all these cases, the sound pressure level and its frequency distribution at measuring points in the above-mentioned areas are determined. For certain measuring points, an experimental measurement of the sound level was performed to verify the numerical results.

Figure 7 presents the noise level in $\mathrm{dB}$, at height points of $1.5 \mathrm{~m}$, at different distances from the back wall of the new acoustic barrier. The results are in octaves in the range $63 \mathrm{~Hz}-4 \mathrm{kHz}$, and the equivalent level for these ranges.

From the results, it can be seen that by varying the distance behind the barrier, the sound pressure level changes differently for the different frequency ranges, and the variations are not unambiguous in direction, character and size. It is visible that at frequencies between $1 \mathrm{kHz}$ and $4 \mathrm{kHz}$ the magnitude of variations reaches $100 \%$, while at low frequencies these changes are up to $30 \%$. This change nature can be explained by the presence of alternating zones of lower and higher acoustic pressure due to the Sonic Crystals effect. The change in the equivalent sound level in this case is below $8 \%$.

Figure 8 gives the sound pressure level in $\mathrm{dB}$ at measuring points at a height of $1 \mathrm{~m}$ between the noise source and the new acoustic barrier. The results are in octaves in the range of $63 \mathrm{~Hz}-4 \mathrm{kHz}$, and the equivalent level for these ranges.

From the results it can be seen that with the variation of the distance between the source and the barrier, the sound pressure level changes significantly only in some of the low frequency ranges, and the variations are not unambiguous in the direction, character and size. The magnitude of these changes is up to $30 \%$. The change in the equivalent sound level in this case is below $7 \%$.

Engineering Sciences, LVII, 2020, No. 1 

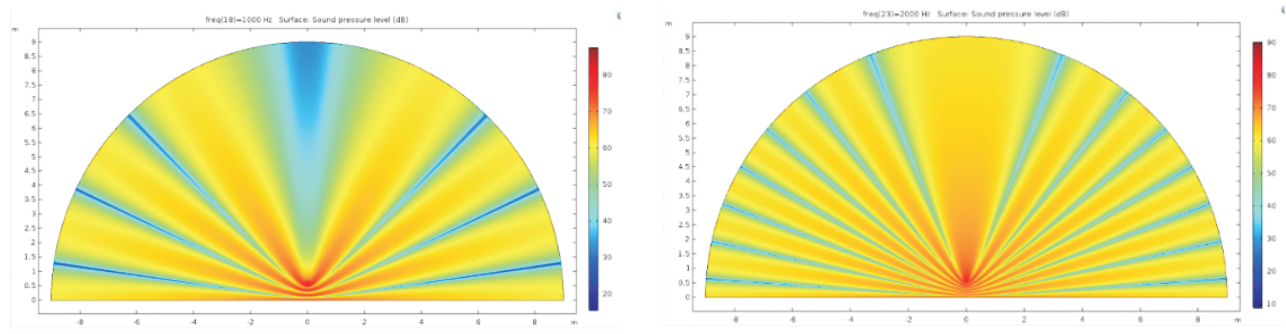

Fig. 5. Source noise level distribution without barrier at frequency octave bands $1 \mathrm{kHz}$ and $2 \mathrm{kHz}$

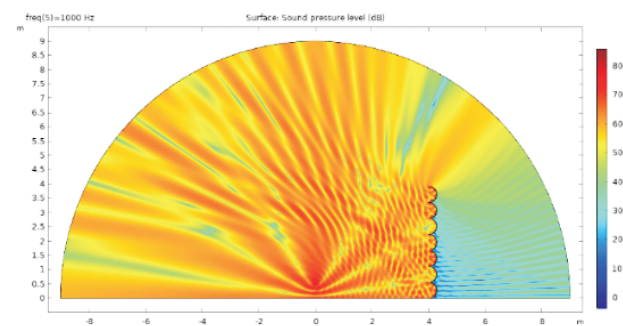

(a)

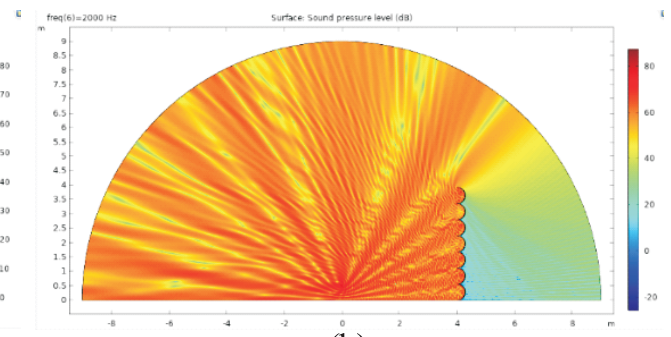

(b)

Fig. 6. Noise level distribution with barrier at frequency octave band: (a) $1000 \mathrm{~Hz}$; (b) $2000 \mathrm{~Hz}$

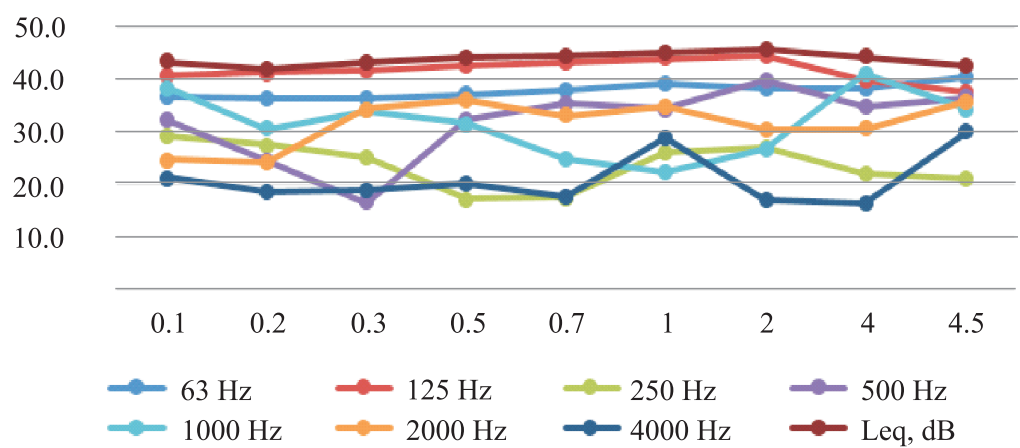

Fig. 7. Sound pressure level at measuring points at a height of $1.5 \mathrm{~m}$ behind the new acoustic barrier, $\mathrm{dB}$

Figure 9 presents the results for the sound pressure level distribution in $\mathrm{dB}$ at measuring points at $0.1 \mathrm{~m}$ distance from the front wall of the barrier. The results are in octaves in the range $63 \mathrm{~Hz}-4 \mathrm{kHz}$, and the equivalent level for these ranges. 


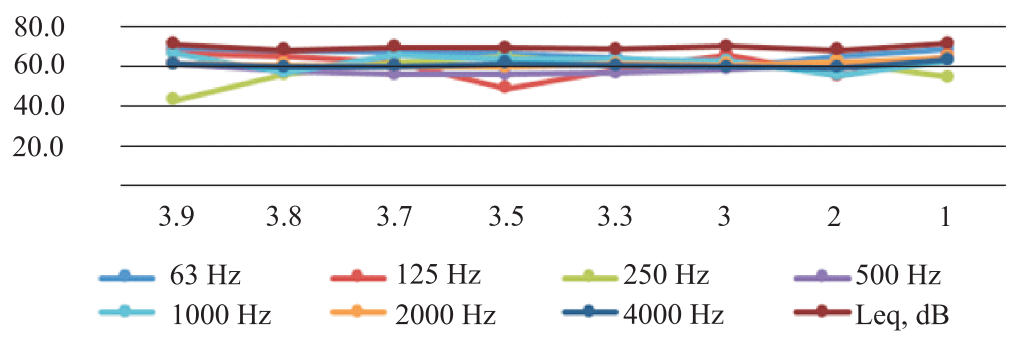

Fig. 8. Sound pressure level at measuring points at a height of $1 \mathrm{~m}$ between the noise source and the new acoustic barrier, $\mathrm{dB}$

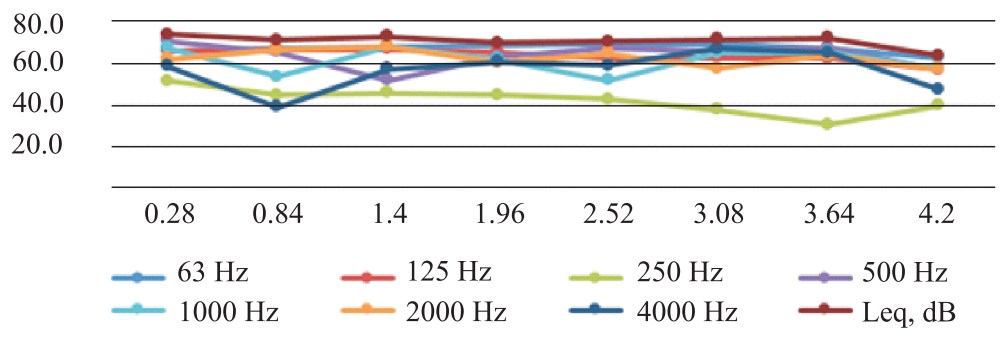

Fig. 9. Vertical sound pressure level distribution at $0.1 \mathrm{~m}$ distance from the front wall of the new acoustic barrier, $\mathrm{dB}$

From the results it can be seen that by varying the height of the measuring point located close to the profile in front of the barrier, the sound pressure level differs substantially for the different octaves, as well as varying in size and direction for different frequency ranges. The magnitude of these variations reaches $100 \%$. The change in the equivalent sound level in this case is up to $30 \%$. Generally, there is a tendency for a slight decrease of the sound level in height.

The following conclusions can be defined:

- The level of sound energy in the space between the source and the barrier is relatively high and close to that of the source;

- The distribution of the sound energy level in the space between the source and the barrier is influenced significantly depending on the frequency, position and parameters of the barrier;

- There is no unambiguous dependence for determining the spatial and frequency distribution of sound energy near the barrier;

- Due to the relatively high equivalent noise levels near the barrier from the source side, it makes sense to develop and integrate power supply devices from transport noise; 
- Synthesis and integration into the new type of barrier of energy production from traffic noise must be done along with the synthesis of the barrier in order to read the frequency distribution of the noise, the acoustic characteristics of the barrier as well as the technological, operational and installation requirements.

\section{CONCLUSION}

In this study it is presented a passive transport noise barrier having areas of high noise level for acoustic energy harvesting. The reflected noise level is done by appropriate design of the barrier profile performing source and reflected acoustic waves interference in small volumes near the barriers. A numerical model and analysis were made to estimate the presence of high-level sound energy areas for implementing the noise-electricity conversion solutions.

The results show presence of high-level sound-energy areas near the barrier for implementing the noise-to-electricity conversion solutions. To fulfill the requirements for barrier high acoustic efficiency and sound energy harvesting solutions, the frequency distribution of the noise and the acoustic characteristics of the barrier materials and geometry must be used.

\section{ACKNOWLEDGEMENTS}

This work was supported by the Bulgarian Ministry of Education and Science under the National Research Programme "Low carbon energy for transport and live", approved by DCM \# 577/17.08.2018.

\section{REFERENCES}

[1] N. Nikolov, D. Benov, And I. Shubin, Acoustic Design of Transport Noise Insulating Barriers, Sofia, ACMO Academic Press (2014) 241 (in Bulgarian).

[2] Patent No UD 96/16230 - 30.05.1996.

[3] Patent No US No 6305492 B1/23.10.2001.

[4] Patent No US 7789193 B2/07.09.2010.

[5] Patent No AT 513615 A4 2014-06-15.

[6] Patent No 89203035.4/29.11.1989.

[7] Patent No DE 4220547 A1/07.01.1993. 
[8] Patent No 1020142017 767.7/10.03.2016.

[9] J. Trevor and P. D'Antonio, Acoustic Absorbers and Diffusers, Taylor \& Francis (2009).

[10] D. J. Thompson, Railway Noise and Vibration, Elsevier (2009).

[11] E. Gieva, I. Ruskova, K. Nedelchev, and I. Kralov, An Investigation of the Influence of the Geometrical Parameters of a Passive Traffic Noise Barrier upon the Noise Reduction Response, in: AIP Conference Proceedings, 2018, 2048, 020020.

[12] M. Aleksandrova, Spray Deposition of Piezoelectric Polymer on Plastic Substrate for Vibrational Harvesting and Force Sensing Applications, AIMS Mat. Sci. (2018) 5 (6) 1214-1222.

[13] I. Kralov and K. Nedelchev, Lowering the Noise Level in the Transport Flows Through Reduction of the Traffic Barrier Reflected Noise, IOP Conf. Ser.: Mater. Sci. Eng. (2019) 618 012051, DOI:10.1088/1757-899X/618/1/012051.

[14] I. Kralov, New Solution for Transport and Industrial Noise Protection through Reflective Noise Barriers, BulTrans-2017, Sozopol, Matec, 2017, 133, DOI: https://doi.org/10.1051/matecconf/201713306001.

[15] M. Yuan, Z. CaO, J. Luo, and X. Chou, Recent Developments of Acoustic Energy Harvesting: A Review, Micromachines (2019) 10 48, DOI:10.3390/mi10010048.

[16] Y. Wang, X. Zhu, T. Zhang, S. Bano, H. Pan, L. Qi, Z. Zhang, and Y. YuAN, A Renewable Low-frequency Acoustic Energy Harvesting Noise Barrier for High-speed Railways Using a Helmholtz Resonator and a PVDF Film, Applied Energy (2018) 230 52-61, https://doi.org/10.1016/j.apenergy.2018.08.080.

[17] R. Monthéard, S. Carbonne, M. Bafleur, V. Boitier, J. Dilhac, et al., Proof of Concept of Energy Harvesting from Aero Acoustic Noise, in: $12^{\text {th }}$ International Conference on Micro- and Nanotechnology for Power Generation and Energy Conversion Applications (PowerMEMS 2012), Atlanta, United States, Dec. 2012, pp. 267-270.

[18] I. Kralov, S. Terzieva, and I. Ignatov, Analysis of Methods and MeMS for Acoustic Energy Harvesting with Application in Railway Noise Reduction, in: Proceedings of MECAHITECH'11, Bucharest, 2011, 3, pp. 56-62.

[19] Y. Ivanova, V. Vassilev, P. Djondjorov, and S. Djoumaliskky, Experimental-Theoretical Approach to the Identification of Effective Sound Attenuation Panels from Recycled Materials, J. Bul. Chem. Comm. (2015) 42 1-8.

[20] H. NoH, Acoustic Energy Harvesting Using Piezoelectric Generator for Railway Environmental Noise, Advances in Mechanical Engineering (2018) 10 (7) 1-9, (c) The Author(s) 2018, DOI: 10.1177/1687814018785058. 
[21] N. Cui, X. Jia, A. Lin, J. Liu, S. Bai, L. Zhang, Y. Qin, R. Yang, F. Zhouc, ANd Y. LI, Piezoelectric Nanofiber/Polymer Composite Membrane for Noise Harvesting and Active Acoustic Wave Detection, Nanoscale Adv. (2019) 1 4909-4914, https://doi.org/10.1039/C9NA00484J;

[22] F. Khan and I. Syed, Hybrid Acoustic Energy Harvesting Using Combined Electromagnetic and Piezoelectric Conversion, Review of Scientific Instruments (2016) 87 025003, https://doi.org/10.1063/1.4941840.

[23] M. Jin, B. LiAng, J. YAng, et al., Ultrathin Planar Metasurface-based Acoustic Energy Harvester with Deep Subwavelength Thickness and Mechanical Rigidity, Sci. Rep. (2019) 9 11152, https://doi.org/10.1038/s41598-019-47649-9

[24] Wu Shao-Hua, Du Li-Dong, Kong De-Yi, et al., Hybrid Device for Acoustic Noise Reduction and Energy Harvesting Based on a Silicon Micro-Perforated Panel Structure, Chin. Phys. B (2014) 23 (4) 044302.

[25] Hu. Shan, Carbon Nanotube Thin Films for Active Noise Cancellation, Solar Energy Harvesting, and Energy Storage in Building Windows, Retrieved from the University of Minnesota Digital Conservancy (2014), http://hdl.handle.net/11299/165421.

[26] Y. A. Farghaly, F. A. A. Hemeida, and S. Salah, Noise Utilization as an Approach for Reducing Energy Consumption in Street Lighting, PLoS ONE (2019) 14 (7) e0219373, https://doi.org/10.1371/journal.pone.0219373.

[27] R. Monthéard, M. Bafleur, V. Boitier, X. Dollat, N. Nolhier, E. Piot, C. Airiau, and J. Dilhac, Coupling Supercapacitors and Aeroacoustic Energy Harvesting for Autonomous Wireless Sensing in Aeronautics Applications, Energy Harvesting and Systems (2016) 3 (4) 1-12, ISSN 2329-8774.

Received March 31, 2020 